\section{AL-AZHAR Dental Journal}

F o r

G i r
The Official Publication

of The Faculty of Dental

Medicine For Girls,

Al-Azhar University Cairo, Egypt.

Print ISSN 2537-0308 • Online ISSN 2537-0316

ADJ-for Girls, Vol. 6, No. 1, January (2019) — PP. 85:93

\title{
Comparison between Two Different Impression Techniques in Ball and Socket Attachments Supported Complete Implant Overdentures on Electromyographic Activity
}

\author{
Marwa S. Seif El Nasr ${ }^{(1)}$, Mostafa M. Abd El Ghany ${ }^{(2)}$ and Dina M. Kholief ${ }^{(3)}$
}

Codex : 12/1901

azhardentj@azhar.edu.eg

http://adjg.journals.ekb.eg

DOI: 10.21608/adjg.2019.6053.1047

\begin{abstract}
Purpose: The purpose of this study was to compare between two different impression techniques in ball and socket attachments supported complete implant overdentures on electromyographic activity. Material and Methods: Ten fully edentulous patients were chosen from the patient clinic of removable prosthodontics department, Patients were divided into 2 groups according to the type of impression technique Group 1: The patients received the denture with the open impression tray technique then a washing period of two weeks elapsed and then switched to the other denture with closed impression tray technique. Group 2: The patients received the denture with the closed impression tray technique then washing period of two weeks elapsed and then switched to the other denture with open tray impression technique. Electromyographic activity measurements were made when the patient in each group wear dentures for one month then a wash out period for two weeks and once more measurements were made when the patient wear the other denture respectively. Masticatory efficiency and electromyographic activity were tested. Results: Patients received dentures made with closed tray impression techniques showed higher masticatory efficiency and lower electromyographic activity than patients received dentures with open tray impression techniques during chewing hard food so significant difference between two techniques were calculated. Conclusions: From the findings of this study; it was concluded that: 1-Masticatory efficiency improved and also the electromyographic activity decreased in patients using closed tray impression technique than that with open tray impression technique during chewing hard food.
\end{abstract}

\section{INTRODUCTION}

Conventional denture wearers frequently report problems with oral function, typically caused by retention and stability problems of the prosthesis. Masticatory function of these subjects is quite poor in comparison with that of healthy dentate patients. They need up to seven times more chewing strokes than patients with a complete natural den-

1. Dentist, New Cairo Hospital, Interior Ministry.

2. Professor of Removable Prosthodontics, Dean of Faculty of Dental Medicine for Girls, Al Azhar University.

3. Assistant Professor of Removable Prosthodontics, Faculty of Dental Medicine for Girls, Al-Azhar University. 
tition to reduce the food to half of original particle $\operatorname{size}^{(1)}$.

An implant supported overdenture was considered as the best choices especially in the lower denture due to Lack of sufficient retention of the complete mandibular denture, especially when compared with the maximum retention obtained with the maxillary denture. Patients with implant retained overdentures have a substantially better masticatory performance and chewing experience and significantly higher unilateral and bilateral maximum bite forces than complete denture wearers. The advent of endosseous implants has greatly reduced the need for much pre-prosthetic surgery such as vestibuloplasty, genial tubercle reduction, and/or floor mouth lowering ${ }^{(2)}$.

Edentulous patients who suffer from the results of extreme residual bone resorption and who experience maladaptive characteristics with mandibular dentures will benefit from implant supported or retained prosthesis. Some authors advocate the use of implant-supported mandibular overdentures as the first choice of treatment for the edentulous mandible, and in addition, the dental literature has shown this treatment to be effective in randomized clinical studies ${ }^{(3)}$.

Restoration of masticatory function is one of the main purposes of dental practice, but its evaluation is mostly depended on subjective view. Many evaluations of masticatory function have been attempted, such as analysis of masticatory performance, occlusal strength, masseter muscular activities and masticatory movement ${ }^{(4)}$.

The impression techniques in implant supported overdentures may influence the retention and stability and thus the oral function of the prosthesis. The accurate record of the implant ,tissue borne material would affect the accurate recording of dental replica to the final prosthesis thus the patient masticatory performance and satisfaction increased ${ }^{(5)}$.
Distortion that occurs during impression making procedure ultimately results in prosthesis that does not seat inertly onto implant abutments. Distortion is defined as the relative movement of a single point or group points away from some original specified reference position such that permanent distortion is apparent. The distortion of an impression material beyond its elastic variety may cause permanent deformation with incorrect impression. An arbitrary 0.4 deformation has been estimated to be clinically significant distortion limit. Distortion during impression procedure may result from dimensional changes of impression material, as well as movement of impression copings within the impression during tightening of the laboratory analogs ${ }^{(6)}$.

Depending on the technique used, the tray will be closed or open above the implant site. In the direct transfer impression technique it will be open by making window above the implant sites to permit the long guide pins (fixation screws) to protrude through the top of the tray. In the direct transfer impression technique the window should be sufficiently large to accommodate the transfer copings and there must be an adequately wide clearance between the copings and the side of the tray. Additional spacing should be provided if the copings are splinted together, as the splinting material may interfere with proper seating of the impression tray unless provision has been made for it ${ }^{(7)}$.

\section{MATERIAL AND METHODS}

\section{Patient selection}

Ten completely edentulous patients were selected from the outpatient Clinic of Removable prosthodontic Department, faculty of dental medicine for girls, Al Azhar University according to special inclusion and exclusion criteria.

\section{Construction of radiographic templates}

A primary impression was made and a stone cast was poured so as to construct a radiographic tem- 
plate. Circular holes $1 \mathrm{~mm}$ depth at canine space on both sides of the cast using round burs were done. The radiographic template was changed to accommodate metal balls at the canine regions by getting ready 2 recesses 2 metal standardized marking spheres of five millimeter in diameter wherever sunk into the holes at the crest of the ridge at every implant potential site and then 0.02 inch gauge, clear acrylic material template was molded incorporating the spheres within it. Radiographic assessment was performed using Cone beam computerized tomography for every patient with the radiographic model within the patient mouth.

\section{SURGICAL PROCEDURES:}

A- Surgical Stent: The metal balls and holes were trained at the planned implant sites (canine regions) to be used as a surgical stent. The stent was modified by reducing the occlusal and labial surfaces at the corresponding implant sites departure the lingual surface intact.

B- Patient preparation: Prophylactic antibiotic coverage was given one hour before every operation. Chlorohexidine mouth wash accustomed disinfects the surgical field and reduce the liability of post-operative infection.

C- 1st stage surgery: The surgical operation was started with an intra-oral crestal incision amongst the two bicuspids and mucoperiosteal flaps were raised each buccally and lingually to show the bone, the implant sockets were ready and also the implants were placed vertically parallel to each other. Titanium implants were used (Multisystem dental implant). Covering screws were screwed to the fixtures. The mucoperiosteal flaps were sutured to permit healing. Special attention was given to flap adaptation of the suture margins. The remaining sutures were detached at two weeks. Standardized oral hygiene instructions were recommended at two weeks postoperatively then reinforced at recall appointments. A healing period of 3 months was permitted to assure complete implant bone osseointegration.

D- Post-surgical instructions: Apply further oral cold fomentations on the lower jaw each twenty minutes, on and off, on the first week. A soft diet was recommended, analgesics as required for pain management, and antibiotic coverage as previously described throughout 1st week. Strictly suits the prescribed medication.

\section{Patients grouping:}

The ten patients were divided into 2 groups (Five patients every group).

Group 1: patients received maxillary complete denture and 2 mandibular lower dentures (The 1st mandibular denture was made beginning with open tray impression technique and also the second denture was made to the same patient using closed tray impression technique) the patient wear the primary denture for one month then a period of two weeks washing period paseds on and then the patient wear the second denture. Electromyographic activity and masticatory efficiency measurements were made when one month that the patient wears every denture.

Group 2: patients received maxillary complete denture and 2 mandibular lower dentures (The $1^{\text {st }}$ mandibular denture was made beginning with closed tray impression technique, the second denture was made to the same patient using open tray impression technique) and also the patient wear the primary denture for one month then a period of two weeksl washing period pass on and then the patient wear the second denture. As in group one electromyographic activity and masticatory efficiency measurements were made when one month that the patient wears each denture.

\section{Prosthetic procedures (Denture construction)}

New conventional denture in the mandible and maxilla were made. The dentures were made in centric relation with balanced articulation and anatomically shaped acrylic teeth. 
A) Impression making: The next procedure was complete for both types of techniques open and closed techniques. a film of the tray adhesive supplied by the manufacturer of the impression material was applied on the boundary of the tray. Polyether impressions (medium consistency) were made following manufacturer's directions. The impression material was mixed using the automatic mixing device. The impression material was applied around the impression coping and also the tray were loaded with the impression material for both technique. The transfer coping was burdened by the polyether syringe and also the loaded impression tray was established over the transfer coping in patient mouth, the tray was completely seated using finger pressure, in case of open impression technique the open transfer coping was seen from the window made in tray,the impression was permitted to set for twelve minutes, that was double the suggested setting time, from the start of merger time at room temperature.

Open tray impression procedures: The implant area on cast was smooth by 2 layers of baseplate wax to allow uniform thickness of impression material. The trays were perforated in the region wherever implants were placed to supply access for the pick up copings. The square impression coping was placed into the implant. The guide screw was tightened using the screwdriver. The heavy consistency polyether impression material was loaded within the impression tray and light consistency polyether impression material was meticulously syringed around the impression copings. The screw driver was used to loosen the guide screw within the impression post. The impression was taken out from patients' mouth. The equivalent analog was selected and also the impression post was placed into the implant analog and also the guided screw was tightened by using the screwdriver. Before screwing in, implant analog should be put in line with the grooves of impression coping to achieve correct passively fitting prosthesis. The impression was now ready to be used to create a mode(fig.1).

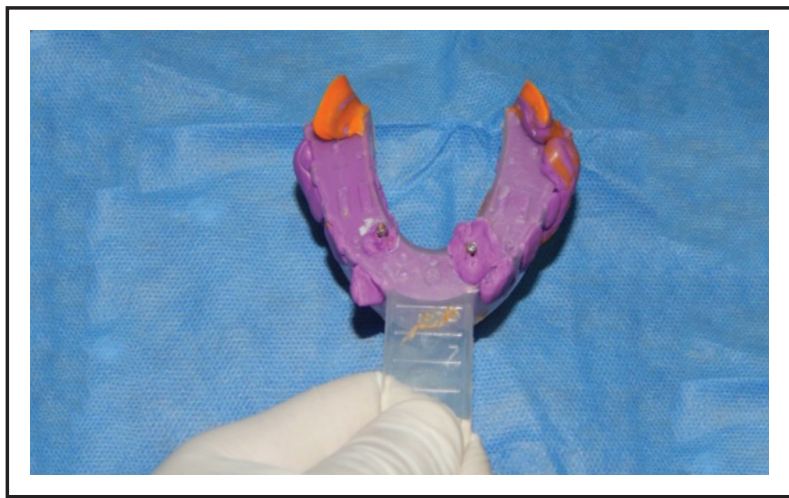

Fig. (1) Open tray impression by using non hexed pick up transfer coping (transfer per pick up h $13 \mathrm{~mm}$ )

Closed tray impression procedures: Impression coping was placed in the implant body guided by the direction of the hex. A radiograph was taken to be sure that the impression coping was completely seated. Light body impression material was injected around impression coping. The impression tray full of a firm impression material was seated. Once impression material was absolutely set, tray was removed. Impression coping from implant with manual screwdriver was removed. Healing abutment was immediately replaced. Patient was then discharged. the fit analog was connected to the impression coping. Impression coping was reinserted into the impression so as to align perfectly, the impression was then sent to the dental laboratory. Two impressions were made for every patient (fig. 2).

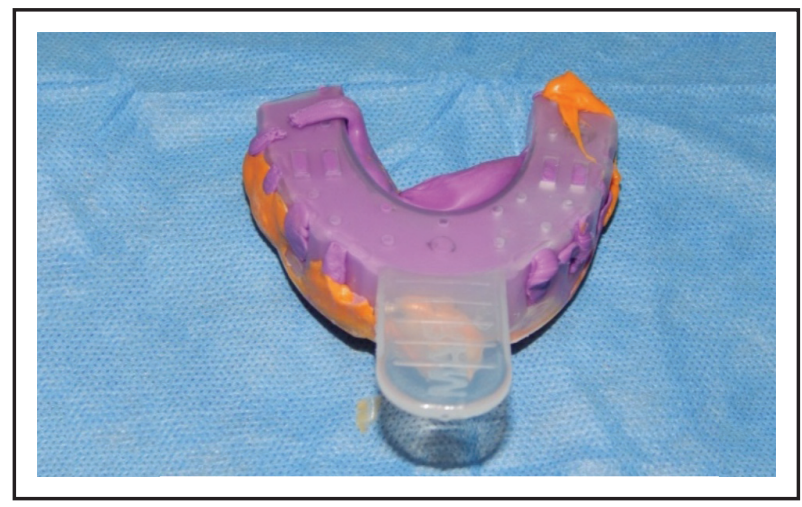

Fig. (2) Closed tray impression by using hexed transfer coping (transfer standard h.9 mm) 
B) Fabrication of occlusal rims: Occlusal rims were made-up and inserted within the patient's mouth to test lip support and labial fullness.

C) Recording jaw relation using face bow record: The patient was seated with his head upright and supported by the headrest. A point was marked $13 \mathrm{~mm}$ in front of auditory meatus on a line running from the outer canthus to the superior border of the tragus. Guidelines for anterior teeth placement were marked on the maxillary occlusal rim. A notch index about $2 \mathrm{~mm}$ deep was made in the first molar region to help positioning the face bow. The mandibular occlusal rim was placed in the patient's mouth and reduced such that sufficient space was available between the two rims to accommodate the bite fork. The U- shaped frame of the face bow along with the condylar rods was placed on the posterior reference points then the condylar rods were locked to the U- shaped frame. Wax was softened shaped to the form of a horseshoe. The bite fork was embedded into this soft wax. A thin layer of petroleum jelly was applied on both the occlusal rims to allow easy separation. Both the occlusal rims and the bite fork with the wax were inserted into the patient's mouth. The patient was asked to close his mouth till both the occlusal rims were embedded into the bite fork. The stem of the bite fork was locked to the transverse rod of the face bow. The entire face bow assembly along with occlusal rims were removed from the mouth and transferred in the articulator.

D) Mounting denture base on the articulator and teeth selection: The anterior teeth were arranged on the occlusal rims using standard principle till acceptable results were obtained. The occlusal rims were transferred to the articulator. The incisal table was rotated till it contacts the incisal pin. The occlusal rims were transferred to the articulator. And the same movements were carried out. The maxillary cast was attached to the articulator using the face bow transfer. The face bow with its bite fork attached to the maxillary occlusal rim was positioned in the articulator. Then fixed with thick mix of dental plaster, Then the mandibular cast was mounted to the articulator after recording the vertical and centric jaw relations, and then attached to the lower member of the articulator using dental plaster. Mounting was useful for detecting the degree of inter-arch space, the relation between both arches and the space available to accommodate attachments. When the casts were articulated during denture fabrication procedure the thickness of the mandibular denture was evaluated. There should had been at least $2-5 \mathrm{~mm}$ of acrylic resin over each abutment. The denture base must was thick enough to minimize the potential for fracture of the denture. Setting up of acrylic teeth with anatomic occlusal surface and suitable teeth size, shape and shade was carried out.

E) Try in: Try-in for waxed up denture was done to test adaptation, denture extension, retention and stability of base plate. Verify jaw relation and vertical height, verify esthetics, check phonetics, and examine waxing -up, so, patient became satisfied with the waxed up denture.

F) Ball and socket attachment application: The suitable ball abutment was selected according to the soft tissue heightthat the collar of the O-ring abutment was a minimum of one millimeter on top of the peri-implant soft tissues. The covering screws were removed from the implants, then ball abutments were screwed to the implants. Metal cups or sockets (female part) were snapped into the ball abutments, then using round bur of appropriate size, an area was created within the fitting surface of the overdenture apposite every of the 2 cups (sockets). The denture was checked within the patient mouth to make sure that there was no interference between the attachment and also the denture. The undercuts below the attachments were blocked using soft block out material (wax). Auto-polymerizing acrylic was placed into the space created within the denture base (fitting surface). The denture was inserted into the patient mouth and was pressed in place, the cup housings were picked into the base 
of the denture. Once the acrylic had set, the denture was far from the patient's mouth, inspected and any voids around the sockets were filled with additional self cured acrylic. Excess material was removed and also the area was finished with an appropriate bur.

\section{Analysis of masticatory efficiency:}

One month following the denture insertion, masticatory efficiency tests were carried out. Masticatory efficiency was evaluated through measures recorded whereas patient was chewing standardized items of food. They were one $\mathrm{cm}^{3}$ of banana and one $\mathrm{cm}^{3}$ of carrot. The at one time mentioned foods represent different types together with soft and hard food respectively. Patient was sitting in an upright position and instructed to chew the food at a traditional rate and swallow pieces of food as he would ordinarily. Four measurements were recorded throughout chew food specimens number of chewing strokes up to the first swallow, number of chewing strokes till the patient's mouth became clear of food, time up to the first swallow and time till the patient's mouth became free of food. 2 investigators were recording the measurements throughout the study independently. One was recording the number of chew strokes and also the other investigator was recording the time elapsed. The patient chewed one piece of every food for 5 times. The mean of the 5 recordings was considered the masticatory performance parameter for that patient.

\section{Electromyographic activity measurement:}

Electromyographic activities measurements of muscles of chew were performed. The measurements were done by 2 of the investigators independently, and in cases with difference of $0.5 \mathrm{~mm}$ or less between the measurements, the mean was used. In cases of variations larger than 0.5 millimeter the myographs were re-examined by each investigators. Surface electromyographic records were obtained from right and left masseter and right and left temporalis muscles by using an electromyographic machine. The set used included a stimulator, scope, amplifier, filter and gain. Electrodes transmitted signals to the main unit. The signals were amplified, filtered and displayed on the scope then printed on paper. The electrodes were silver chloride disc electrodes soldered into a conducting wire. The conductor placement was determined by instructing the patient for firm clenching of his denture teeth, so making a vicinity of facial muscle contraction. Alcohol swab was used for cleaning skin to decrease skin resistance to electrical connection. Electrode gel was applied to skin for rising conductivity. One in all the masseter muscle electrodes were placed one $\mathrm{cm}$ behind the palpated anterior border within the space of the greatest lateral distension. The opposite conductor was placed caudal to the primary one. one in all the anterior temporal muscle electrodes was placed over its anterior portion at the realm of its greatest lateral distension. The other electrode was placed relative to the primary one. A ground electrode was connected to the patient's forehead. Electrodes were fixed by adhesive tapes. The recordings were obtained throughout the following conditions: chew $1 \mathrm{~cm}^{3}$ of banana that described soft food. Chew $\mathrm{cm}^{3}$ of carrot that described hard food. Impulses were printed by laser printers. Statistical analysis was carried out. Measuring the activity of anterior temporalis muscle and measuring the activity of facial muscle.

\section{Statistical analysis:}

All the measured information was tabulated and statistically analyzed. Values were given as mean and variance (SD) values. Information was explored for normality using Kolmogorov-Smirnov test of normality. The results of Kolmogorov Smirnov test indicated that most of knowledge was normally distributed (parametric data), therefore, independent t test was used to compare between groups. The significance level was set at p \&lt; 0.05. Statistical analysis was performed with SPSS 18.0 (Statistical Package for Scientific Studies, SPSS, Inc., Chicago, IL, USA) for Windows. 


\section{RESULTS}

Regarding masticatory efficiency of muscles of mastication according to type of impression technique relating to number of strokes during chewing soft food: relating to 1 st swallow, the greatest mean was recorded in open tray impression technique. Independent $t$ test revealed no statistically vital distinction between each impression techniques ( $\mathrm{p}=0.386)$. Regarding mouth clearance, the greatest mean was recorded in open tray impression technique. Independent $t$ test at unconcealed no statistically vital distinction between each impressions.

Regarding masticatory efficiency of muscles of mastication according to type of impression technique relating to number of strokes during chewing hard food: relating to 1st swallow, the greatest mean was recorded in open tray impression technique independent $t$ test revealed a statistically vital difference between each techniques $(\mathrm{P}=0.013)$. Relating to mouth clearance, the greatest mean was recorded in open tray impression technique $(19.67 \pm 1.08)$. Independent $t$ test revealed a statistically vital difference between each technique. b- Time of swallowing:

Regarding masticatory efficiency of muscles of mastication according to type of impression technique relating to time of swallowing during chewing soft food: regarding 1st swallow, the greatest mean was recorded the greatest mean was recorded in open tray impression technique. Independent t test revealed no statistically vital difference be- tween each techniques $(\mathrm{P}=0.075)$. Regarding mouth clearance, the greatest mean was recorded the greatest mean was recorded in open tray impression technique. Independent $t$ test unconcealed no statistically vital difference between each technique.

Regarding masticatory efficiency of muscles of mastication according to type of impression technique relating to time of swallowing during chewing hard food: relating to 1 st swallow, the greatest mean was recorded the greatest mean was recorded in open tray impression technique. Independent $t$ test revealed a statistically vital difference between each techniques ( $\mathrm{P}=0.044)$. Relating to mouth clearance, the greatest mean was recorded the greatest mean was recorded in open tray impression technique. Independent $t$ test revealed a statistically significant difference between both techniques $(\mathrm{P}=0.032)$.

Regarding electromyographic activity of masseter muscle according to type of impression technique during chewing soft food: With soft food, the greatest mean value was recorded in open tray impression technique. Independent $t$ test revealed non-significant difference between each techniques $(\mathrm{P}=0.535)$.

Regarding electromyographic activity of masseter muscle according to type of impression technique during chewing hard food: With hard food, the greatest mean was recorded in open tray impression technique. Independent $t$ test revealed a significant difference between both techniques $(\mathrm{P}=0.042)$ (table 1),

Table (1) Electromyographic activity of masseter muscle according to type of impression technique during chewing hard food

\begin{tabular}{|c|c|c|c|c|c|c|c|c|}
\hline \multicolumn{2}{|c|}{ Impression technique } & Mean & Std. Deviation & Std. Error & Min & Max & $\mathrm{t}$ & $\mathrm{P}$ \\
\hline & - Closed & 236.19 & 27.09 & 11.06 & 202.04 & 354 & 2.33 & $0.042 *$ \\
\hline & - Opened & 275.91 & 31.79 & 12.98 & 196.17 & 377.41 & & \\
\hline
\end{tabular}

Significance level $p<0.05$, *significant 
Table (2) Electromyographic activity of temporalis muscle according to type of impression technique during chewing hard food

\begin{tabular}{|l|l|c|c|c|c|c|c|c|}
\hline \multicolumn{2}{|c|}{ Impression technique } & Mean & Std. Deviation & Std. Error & Min & Max & $\mathrm{t}$ & $\mathrm{P}$ \\
\hline \multicolumn{2}{|l|}{ - Closed } & 211.73 & 72.05 & 29.42 & 106.57 & 308.08 & 2.53 & $0.03 *$ \\
\hline & - Opened & 314.79 & 69.29 & 28.29 & 218.25 & 419.12 & & \\
\hline
\end{tabular}

Significance level $p<0.05$, *significant

Regarding electromyographic activity of temporalis muscle according to type of impression technique during chewing soft food: With soft food, the best mean was recorded in open tray impression technique. Independent $t$ test revealed non- significant difference between both techniques $(\mathrm{P}=0.091)$.

Regarding electromyographic activity of temporalis muscle according to type of impression technique during chewing hard food: With hard food, the greatest mean value was recorded in open tray impression technique. Independent $t$ test revealed a significant difference between both techniques $(\mathrm{P}=0.03)$ (Table 2).

\section{DISCUSSION}

The obtained results stated that closed tray impression techniques had better masticatory efficiency and were more accepted than open tray impression techniques. The current study revealed that masticatory efficiency and electromyographic activity for closed tray were lesser than that in open tray impression techniques, this finding contradicts with results of some pervious researches ${ }^{(8,9)}$.

Some studies do not show significant difference between the two techniques. Some studies stated that closed tray impression techniques record more fine details thus improving masticatory efficiency relating to open tray impression technique in such cases with implants in canine. Some other studies revealed a higher difference in comparing between two techniques in limited mouth opening were it revealed higher masticatory efficiency and lower electromyographic activity regarding to closed impression techniques where it have been more accurate in such cases ${ }^{(10)}$.
Findings that there is no vital distinction between the closed tray technique and unsplinted open receptacle technique were sited. Another studies reported that the closed tray technique yielded a lower electromyographic activity than open tray technique. Most analysis that centered on the techniques of implant impressions in clinical things showed that masticatory effectiveness was significantly higher in closed tray impression technique when compared to open tray impression technique ${ }^{(11)}$.

In Agreement with this study some authors applied two methods of programmed search and manual search in several relevant journals to study articles associated with implant impression, common methods and modifications made. They finally concluded that direct impression (open tray) method gives lower masticatory efficiency than indirect (closed tray) method $^{(12)}$.

Some others have clinically verified both techniques in spite of their different statistical significance and have identified the errors as non-significant. Others had noticed passive relation as one of the prerequisites of implant supported prosthesis. They did not distinguish a significant difference between open tray and closed tray techniques. Also angulations of the implants were not mentioned in their research ${ }^{(13)}$.

Based on a research ; masticatory efficiency and electromyographic activity of two impression techniques, namely open tray and closed tray, were not significantly different. They also noticed that implant angle and implant number were different from average error of angulations but not large enough to be simply interpreted ${ }^{(14)}$. 
Other studies investigated the masticatory efficiency and electromyographic activity of the two impression techniques for single implants and focused on the accuracy of types of two impression processes of implants through direct and indirect methods; using poly ether and poly vinyl siloxane materials.; they demonstrated that casts have been built through closed tray (indirect) method with metallic copings on the surface of the implant were more precise than the casts fabricated by open tray (direct) method with plastic copings ${ }^{(6)}$.

Moreover- masticatory efficiency and electromyographic activity of closed tray technique that was lesser during this study is credited due to its simplicity, accuracy of operator in implement the technique. Relating to the in consistent results in preference of either direct (open tray) or indirect (closed tray) technique, it seems that the accuracy of the impression has been more effective than the other factor in final results ${ }^{(15)}$.

\section{CONCLUSIONS}

From the findings of this study; it was concluded that:

1- Masticatory efficiency improved and also the electromyographic activity decreased in patients using closed tray impression technique than that with open tray impression technique throughout chew hard food.

2- Masticatory efficiency and electromyographic activity weren't affected by changing the impression technique throughout chewing soft food.

\section{REFERENCES}

1. AssuncaoWG,Barao VA, Delben JA, Gomes EA, Tabata LF.A comparison of patient satisfaction between treatment with conventional complete dentures and overdentures in the elderly: A literature review. Gerodonto J 2009; 33: 1-9.

2. Abu AlhaijaES, AlZoubi LA, Al Rousan MA, Hammad MM.Maximum occlusal bite forces in jordanian individuals with different dentofacial vertical skeletal patterns. Euro J Orthodont2005; 32:71-7.
3. Akca k, Eser A, Canay S.Numerical simulation of the effect of time to loading on peri implants bone. Med Engphys J 2011; 32:7-13.

4. Yogesh RA, Pankaj Y, Mariette D, Jagjeesingh S, Jain A.A Report of two cases. J ClinDiagn Res 2013; 7: 96-8.

5. Albrektsson T, Zarb G, Worthington P, Eriksson AR.The long term efficacy of currently used dental implants: a review and proposed criteria of success. Int J Oral Maxillofac 2007; 1:11-25.

6. JolyJC de Lima AF, da Silva RC. Clinical and radiographic evaluation of soft and hard tissue changes around implants: A pilot study. J Periodontol 2003; 74: 97-103.

7. Howerton WB, Mora MA. Advancements in digital imaging: what is new and on horizon? J Am Dent Assoc 2008; 139: 205-45.

8. Sachulze D, Heiland M, Thurmann H, Adam G. Radiation Exposure during midfacial imaging using 4 and 16- slice computed tomography: cone beam computed tomography systems and conventional tomography. DentomaxillofacRadiol 2004:33:83-86.

9. Bornstein MM, Balsiger R, Sendi P, Arx T. Morphology of the nasopalatine canal and dental implant surgery: a radiographic analysis of 100 consecutive patients using limited cone-beam computed tomography. Clin Oral Impl Res 2011; 22:295-301

10. Kamburoglu K, Murat S, Kolsuz E, Kurt H, Yüksel S, Paksoy C. Comparative assessment of subjective image quality of cross-sectional conebeam computed tomography scans. J of Oral Sci 2011; 53: 501-08.

11. Hassan B, Souza PC, Jacobs R, Berti SA, Stelt P. Influence of scanning and reconstruction parameters on quality of three-dimensional surface models of the dental arches from cone beam computed tomography Clin Oral Invest 2010; 14:303-10.

12. Ganz SD. Cone beam computed tomography-assisted treatment planning concepts. Dent Clin N 2011; 55:515-36.

13. Luk LC, Pow EH, Li TK, Chow TW. Comparison of ridge mapping and cone beam computed tomography for planning dental implant therapy. Int J Oral Maxillofac Implants 2011; 26:70-74.

14. Misch CE. Contemporary implant dentistry. 3rd ed. Canada: Mosby Inc, St. Louis 2008. P 38-67, 645-67.

15. Sahin S, Cehreli MC. The significance of passive framework fit in implant prosthodontics: current status. Impl Dent J 2001;10: 85-92. 